\title{
Reduction of Electron Scattering Image Blur for Atmospheric Scanning Electron Microscopy
}

\author{
Yusuke Ominami ${ }^{1}$, Kenji Nakahira ${ }^{2}$, Shinsuke Kawanishi ${ }^{1}$, Sukehiro Ito ${ }^{1}$ \\ ${ }^{1}$ Hitachi High-Technologies Corporation, 882, Ichige, Hitachinka-shi, Ibaraki-ken, 312-8504, Japan \\ ${ }^{2}$ Hitachi Ltd. Yokohama Research Laboratory, 292 Yokohama-shi, Kanagawa, 244-0817, Japan
}

Recently, methods for observing samples under atmospheric pressure in a scanning electron microscope (SEM) have been reported by some investigators. We proposed a novel atmospheric SEM (ASEM) technique for observing samples which are present in ambient air conditions but are separated from the membrane [1]. In our system, the environment around the sample can be kept in ambient air conditions (Fig. 1(a)). While wet materials is clearly observed without direct sample membrane contact at an optimized distance, typical atmospheric SEM image taken in atmosphere is more blurred compared to conventional SEM image taken in vacuum condition. The reason why ASEM images looks like "blurred" is because electron beam is scattered by electron scattering region shown in Fig. 1(b). In order to reduce the electron scattering effect, some methods utilizing light element gas [2] or additional vacuum pump to reduce pressure [1] $\left(10^{4} \sim 10^{5} \mathrm{~Pa}\right)$ have been developed. A typical atmospheric SEM image is shown in Fig. 1(c). Brightness of point B is brighter than that of point A, although the edge of number "9" is clear. The image gives us a consideration that the profile of electron beam arriving at sample is estimated as sum of scattered and un-scattered electrons beam. As a result, the image in Fig. 1(c) seems to be blurred. Based on the consideration, we develop an image enhancement algorism for ASEM (electron scattering corrector: ES-Corrector). By using this algorism, blurring created by scattered electrons in ASEM image can be improved after detection of SEM image.

Figure 2 shows SEM images of $\mathrm{Cu}$ mesh (Fig. 2(a)(b)) taken in atmospheric pressure. Figure 2(c) and (d) are restored images using ES-Corrector. The images show great improvements in clarity and edge sharpness than the observed images. The microstructures on $\mathrm{Cu}$ mesh observed in Fig. 2(c) and (d) are compatible to those in SEM images taken in vacuum Fig. 2(e) and (f). Figure 3 shows SEM images of a filter paper (Fig. 3(a)), renal glomerulus without metal staining (Fig. 2(b)), a leaf surface of the Japanese radish(Fig. 3(c)), and blood cells fixed with $1 \%$ glutaraldehyde and immune-stained with gold particles (Fig. 3(d)) taken in atmospheric pressure at room temperature. Figure 3(a)-(h) is the original and restored images. The images show great improvements in clarity and edge sharpness than the observed images. It has been shown that the ES-Corrector algorism to reduce effect of scattered electrons from ASEM image can improve image quality.

\section{References}

[1] Y. Ominami et al., accepted in Microscopy (2014).

[2] K. Nguyen, M. Holtz, and D. Muller, Microsc. Microanal. 19 (Suppl 2) (2013) 

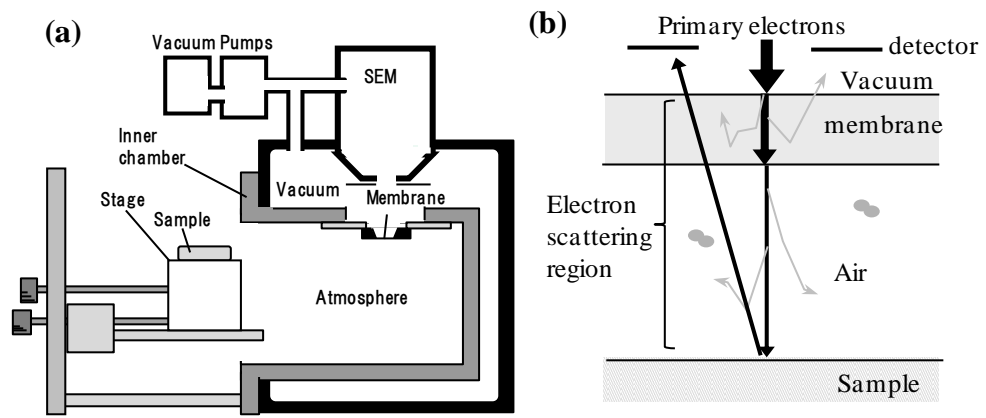

(c)

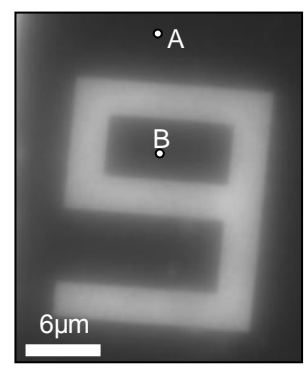

Fig. 1 (a) Schematics of our ASEM. (b)Events of primary electrons. (c)A typical ASEM image.

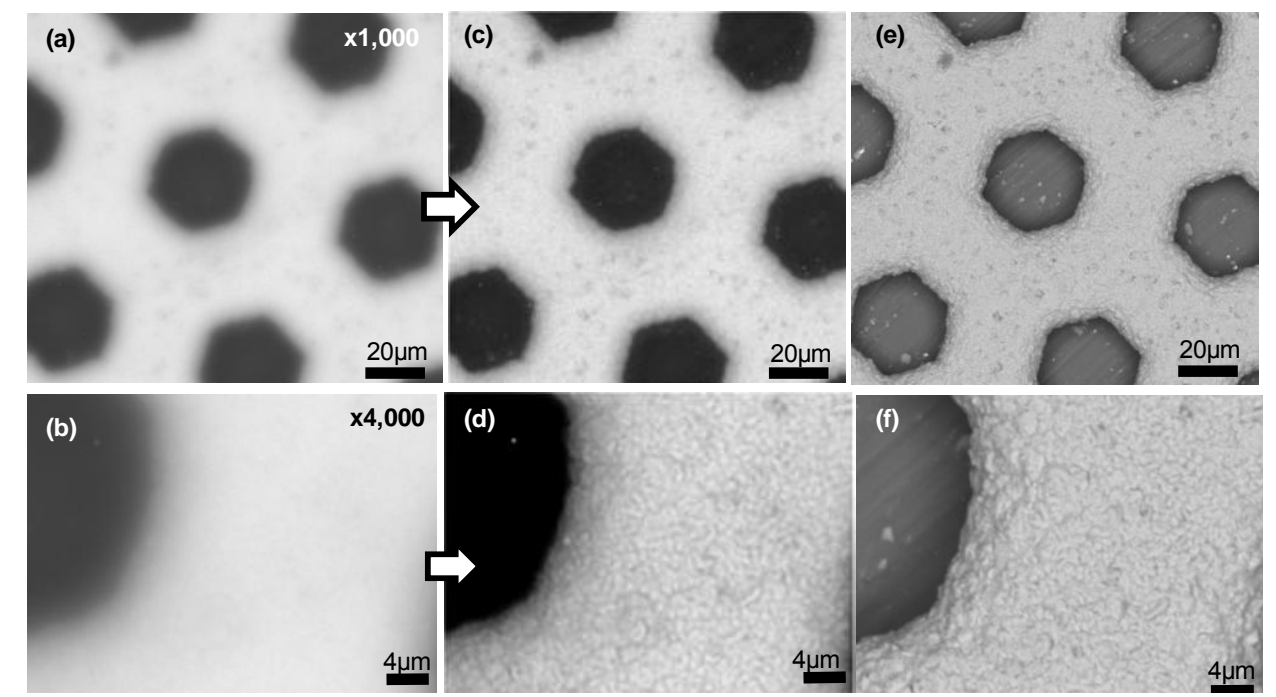

Fig. 2 SEM images of $\mathrm{Cu}$ mesh. Images (a)(b) are taken in atmospheric pressure. Images of (c)(d) are restored images using ES-Corrector. Images of (e)(f) are taken in vacuum condition.
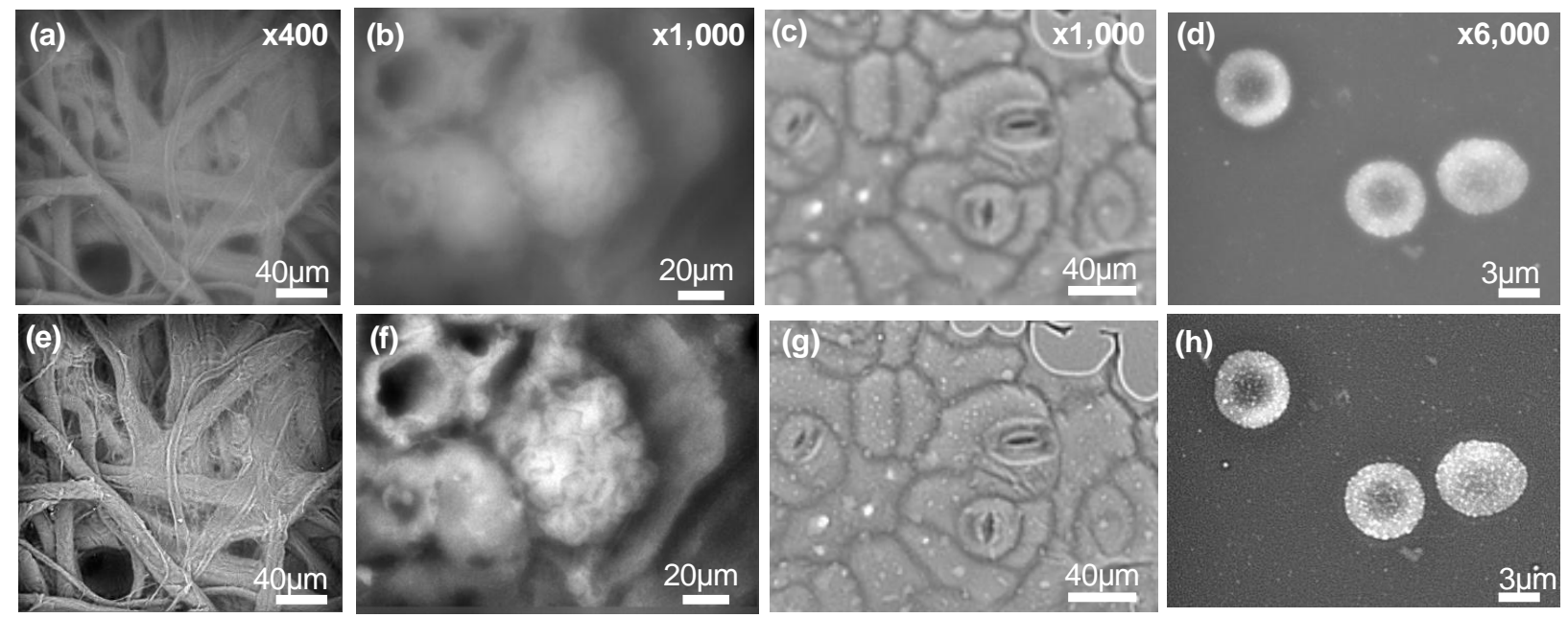

Fig. 3. SEM images taken at 1 atm (a)(e) a filter paper, (b)(f) rat renal glomerulus(un-stained), (c)(g) a leaf surface of Japanese radish, (d)(h) blood cells fixed with $1 \%$ glutaraldehyde and immune-stained with gold particles. $(\mathrm{e})(\mathrm{f})(\mathrm{g})(\mathrm{h})$ are images improved using the developed ES-Corrector. 